\title{
Escritores en la Revolución Cubana Articulaciones entre literatura y política en los años sesenta (1964-1966)
}

\author{
Writers in the Cuban Revolution \\ Articulations between literature and politics in the sixties (1964- \\ 1966)
}

\author{
Leonardo Martín Candiano \\ Instituto de Filología y Letras Hispánicas, \\ Facultad de Filosofía y Letras, \\ Universidad de Buenos Aires (Argentina) \\ leonardocandiano@hotmail.com
}

\begin{abstract}
Resumen
El artículo analiza los posicionamientos de los escritores cubanos insertos en la Revolución respecto de su propio oficio literario. Para ello focaliza en una serie de conversatorios y entrevistas colectivas difundidas en los medios de la Revolución a mediados de los años sesenta, en particular en las revistas Casa de las Américas y Bohemia.

De este modo, se pretenden definir dentro de un período concreto los lineamientos a partir de los cuales los narradores y poetas cubanos asumieron su práctica específica en articulación con su integración en un proceso emancipatorio, lo cual derivó en replanteamientos y quiebres respecto de concepciones tradicionales sobre la producción estética, su autonomía y su vinculación con otras prácticas sociales.

En particular, se inquiere en los textos "Conversación sobre arte y literatura" y "Entrevista", publicados en Casa de las Américas № 22-23 de enero-abril de 1964 (número dedicado a "La nueva literatura cubana"), y en "Literatura revolucionaria", aparecido en Bohemia el 22 de julio de 1966; todo lo cual dialoga críticamente tanto con los rasgos salientes de la literatura cubana del período como con el rumbo asumido entonces por el socialismo cubano.
\end{abstract}

\section{Palabras Clave}

Literatura; Revolución; Casa de las Américas; Intelectualidad; Realismo.

\begin{abstract}
The present article analyses the positioning of Cuban writers immersed in the Revolution regarding their own literary work. For such purpose, this study is focalized in a series of collective conversatories and interviews spread among the media of the Revolution in the middle of the sixties; especially the magazines Casa de la Américas and Bohemia.
\end{abstract}

Esta obra está sujeta a la Licencia Reconocimiento-NoComercial-CompartirIgual 4.0 Internacional de Creative Commons. http://creativecommons.org/licenses/by-nc-sa/4.0/ 


\section{Leonardo Martín Candiano}

In this way, it is intended to define, within a concrete period, the lineaments from which Cuban narrators and poets assumed their specific practice in articulation with their integration into an emancipatory process that derived in reconsiderations and breaks related to traditional conceptions of the aesthetic production, its autonomy and its connection with other social practices.

This work examines, particularly, the texts "Conversación sobre arte y literatura" and "Entrevista", published in Casa de las Américas No 22-23 of January-Abril in 1964 (number dedicated to "La nueva literatura cubana"), and in "Literatura revolucionaria", appeared in Bohemia in July 22nd in 1966. This analysis has a critical dialogue both with the relevant features of the Cuban literature of the period and with the path assumed then by the Cuban socialism.

\section{Keywords}

Literature; Revolution; Casa de las Américas; Intelectuality; Realism.

\section{El surgimiento de un movimiento literario y la gestión de la cultura en Cuba}

Es prolífica la cantidad de estudios sobre Cuba que anclan su mirada en la política cultural de la Revolución durante los años sesenta, (Fernández Retamar, 1967; Walsh, 1968; Rama, 1971; Quintero Herencia, 2002; Gilman, 2003; Rojas, 2006; Martínez Pérez, 2006; Pogolotti, 2006; Kohan, 2006; Guanche, 2006; Gallardo Saborido, 2009; Fornet, A., 2007; Artaraz, 2011, Fornet, J., 2013; Alonso, 2018; entre otros). En ellos, de forma abrumadora se estipula la existencia de un primer período que se inicia con la toma del poder en enero de 1959 y se cierra aproximadamente entre febrero y abril de 1971 con el encarcelamiento de Heberto Padilla y las resoluciones del Congreso Nacional de Educación y Cultura que dieron origen a lo que Ambrosio Fornet denominó Quinquenio Gris (1971-1976), una etapa caracterizada por una mayor rigidez ideológica y por una regimentación de la práctica estética, rasgos que ya se vislumbran con cierta elocuencia desde mediados de 1968 y cuyos primeros antecedentes pueden rastrearse aún con anterioridad.

Por lo tanto, los años en los que se circunscribe nuestro trabajo -1964/1966constituyen un acotado momento dentro de una etapa más extensa, que procura ahondar en fuentes directas muy poco exploradas en búsqueda de un aporte a un mayor esclarecimiento de los debates sobre prácticas, funciones y roles del intelectual en un contexto revolucionario, así como en referencia a la articulación entre cultura y política en la construcción del socialismo en Cuba.

Las fuentes seleccionadas presentan intervenciones realizadas mayoritariamente por escritores integrados al proceso revolucionario. De este modo, se indaga en reflexiones de la intelectualidad cubana en relación a la gestación de una noción de literatura que forme parte de la nueva cultura socialista. Se inquiere, con ello, en los fundamentos desde los que se orientó la realización artística, impartidos por quienes contemporáneamente producían su obra literaria. 


\section{Escritores en la Revolución Cubana. Articulaciones entre literatura y política en los años sesenta (1964-1966)}

Este período de los primeros doce años de la Revolución no fue homogéneo ni unidireccional y estuvo atravesado por continuas fricciones y búsquedas de síntesis. En su interior contrastan coyunturas peculiares con prácticas dominantes diferenciables. Los lapsos más abordados suelen ser el inicial de 1959 a 1961 que marca el fin de la dictadura de Batista y llega hasta la declaración del carácter socialista de la Revolución, y el final de 1968 a 1971 que expresa el comienzo de una etapa de mayor alineamiento con la URSS. Ello fija un tiempo intermedio marcado por una serie de polémicas públicas y por la reconsideración de la función intelectual, al cual un sector de la crítica (Martínez Pérez, Artaraz, Fornet, A., Alonso) desagrega entre el comienzo de una pugna ideológica -que en lo cultural se expresó fundamentalmente en la disputa entre los denominados "herejes" y los llamados “dogmáticos" (1961-1964)- y el auge de una política más independiente de la Revolución respecto de las potencias socialistas (1965-1968) (Guanche) que tuvo en el Salón de Mayo de 1967 y en el Congreso Cultural de La Habana de enero de 1968 sus más elocuentes manifestaciones dentro del plano intelectual.

Roberto Fernández Retamar advirtió que estos momentos si bien son definibles, también se contaminan mutuamente ya que: "no se separan bruscamente ni, en rigor, se extinguen. (...) Acaso podrían presentarse estas etapas como el predominio de unas fuerzas sobre otras, pero no necesariamente como el exterminio de unas u otras" (1967: 13). En el mismo sentido, Alonso sugirió la imposibilidad de aproximarse a la cultura cubana de los sesenta: "fuera de sus contradicciones, de las incertidumbres y los errores e incoherencias" (2018: 84). Por ello, aunque es factible determinar tendencias dominantes, no puede obviarse la concurrencia de otras incluso antagónicas o incompatibles- que se despliegan contemporáneamente, algunas con notoria vehemencia.

Esta complejidad originó conclusiones críticas discordantes sobre la política cultural -y no solamente ella- en Cuba, que enfatizan en aspectos diversos del quehacer intelectual, la práctica estética y el papel de las instituciones creadas por la Revolución, no obstante lo cual los analistas coinciden en subrayar como rasgo característico un redimensionamiento de la cultura desde un plano político que le otorgó un lugar protagónico a los intelectuales y a su obra.

Es extensa la bibliografía que destaca en los inicios de la Revolución Cubana una inédita propagación en la producción de bienes simbólicos sostenida por la promoción de una amplia diversidad estética (Fernández Retamar, Walsh, Rama, Martínez Pérez, Pogolotti, Kohan, Guanche, Artaraz, Alonso). Esto se verifica al comprobar el cobijo otorgado entonces en la isla a variadas tendencias literarias a partir no solamente de difusión en medios oficiales y masivas tiradas editoriales, sino también en los premios de instituciones como Casa de las Américas y la Unión Nacional de Escritores y Artistas de Cuba -UNEAC-.

Esta caracterización es asumida incluso por aquellos en cuya lectura se comprueba un agudo cuestionamiento del proceso revolucionario, como es el caso de Rafael Rojas, quien determina la instauración del socialismo en 1961 como el inicio de un "régimen totalitario" (2006: 11) producto del "ordenamiento comunista" (2006:17) 


\section{Leonardo Martín Candiano}

y proclama que: "Fidel Castro no era más que una réplica de Stalin con oratoria martiana y gestualidad mussolinesca" (2006: 312). Más allá de esta apreciación, parafraseada reiteradamente durante la totalidad de Tumbas sin sosiego, el autor acepta que:

“El nuevo poder de la cultura, en los años 60, siguió una lógica institucional y políticamente distributiva, que permitió una década de intensa creatividad y pluralismo en la literatura, la música, el cine y la plástica cubanas (...) facilitó la emergencia de instituciones tan renovadoras como el ICAIC o Casa de las Américas y de publicaciones tan vivas y polémicas como Lunes de Revolución, El Caimán Barbudo y Pensamiento Crítico" (2006: 449).

La sola enumeración de esas revistas da cuenta de que la extensión temporal de esa amplitud que observa Rojas en la producción artística cubana se desarrolla incluso más allá de la década que enuncia el pasaje citado, pues va desde Lunes de Revolución (1959/1961) a Pensamiento Crítico (1967/1971), cubriendo los doce años de este primer período revolucionario. De allí que aunque la tesis principal que pretende sustentar sea que desde 1961 la presión del aparato cultural estatal habría restringido la libertad estética, señale a la vez que: “...todavía en La Habana de los 60 se podía soñar con una cultura crítica, refinada, que compartiera los valores socialistas de la Revolución" (2006: 312). Similar propuesta se reconoce en Figuraciones del espacio. Letras e imaginario institucional de la Revolución Cubana (1960-1971), de Quintero Herencia -texto que se detiene fundamentalmente en la experiencia de Casa de las Américas-. Estos ensayos, si bien concluyen en la emergencia de un antiintelectualismo cubano que se distingue de las lecturas de Pogolotti, Jorge Fornet, Ambrosio Fornet, Kepa Artaraz, Néstor Kohan y Julio César Guanche ${ }^{1}$, acuerdan con ellos en remarcar la pluralidad en el área cultural. Es que desde su acceso al poder y por lo menos hasta 1971, la Revolución agrupó a dispersos y en ocasiones opuestos grupos intelectuales y le confirió al ámbito cultural una heterogeneidad que se expresó con notoriedad en el terreno estético. Como señala Pogolotti:

\footnotetext{
"El presente reconstituía la tradición del pasado. El ballet y la danza contemporánea se desarrollaban junto al Conjunto Folklórico Nacional. El teatro estrenaba a Brecht y a Lope de Vega. Los libros recogían textos recién salidos del horno y ponían en circulación lo mejor de la herencia literaria venida de todas partes. En las artes plásticas, los salones rendían cuenta de la contemporaneidad y las retrospectivas reconocían la vigencia de los fundadores de la vanguardia. Sin olvidar a los clásicos, la música se lanzaba a la aventura de la experimentación" (X).
}

\footnotetext{
${ }^{1}$ Estos críticos no omiten las tensiones generadas en el área ni la existencia de una serie de acciones que se alejan de la amplitud esgrimida, pero considera que la tendencia dominante en los años sesenta no fue esa, sino la inestable coexistencia de ideas estéticas dentro del proceso revolucionario y la promoción del debate, lo que fundamentan a partir de la explicitación del numeroso caudal de producciones culturales, publicaciones e instituciones originadas entonces.
} 


\section{Escritores en la Revolución Cubana. Articulaciones entre literatura y política en los años sesenta (1964-1966)}

Una derivación de lo señalado fue la promoción de una literatura gestada por autores pertenecientes a camadas intelectuales diferentes, con poéticas particulares distintas e influidos por corrientes contrapuestas. La escritura artística contuvo y propagó prácticas que fueron desde la constitución del Testimonio como género pos-novelesco a partir de la obra de Miguel Barnet -Biografía de un cimarrón (1966)- hasta la experimentación vanguardista de Reinaldo Arenas -Celestino antes del alba (1965)-, pasando por el despliegue definitivo de lo real maravilloso en la novelística de Alejo Carpentier -El siglo de las luces (1962)-. Asimismo, se diseminaron narrativas ligadas a estéticas reflejas como el realismo tradicional en la obra inaugural de José Soler Puig -Bertillón 166 (1960)- o, ya a inicios de los años setenta, mediante la incursión dentro del "realismo socialista" en autores como Manuel Cofiño López -La última mujer y el próximo combate (1971)-. A ello debemos incorporar la aparición de la "narrativa de la violencia" con los cuentos de jóvenes como Jesús Díaz -Los años duros (1966)-, Norberto Fuentes -Condenados de Condado (1968)- y Eduardo Heras León -La guerra tuvo seis nombres (1966), Pasos en la hierba (1971)-, y a aquellos influenciados en parte por el compromiso sartreano, como Guillermo Cabrera Infante -Así en la paz como en la guerra (1960)-, Lisandro Otero -La situación (1963), En ciudad semejante (1970)- y Edmundo Desnoes -No hay problema (1961), Memorias del subdesarrollo (1965)-.

Entre estas obras, las novelas Bertillón 166, La situación y La última mujer y el próximo combate, junto con los volúmenes de cuentos Los años duros y Condenados de Condado, recibieron el premio continental Casa de las Américas en sus rubros, mientras que Los pasos en la hierba obtuvo una mención especial en el mismo concurso. Celestino antes del alba y La guerra tuvo seis nombres, por su parte, fueron galardonados en el premio nacional de la UNEAC.

Aunque no participaron de concursos y por lo tanto no formaron parte de obras premiadas, la difusión masiva de El siglo de las luces, Memorias del subdesarrollo y Así en la paz como en la guerra generó que se convirtieran en íconos de la literatura cubana del período. A modo de ejemplo podemos señalar que el libro de cuentos de Cabrera Infante logró en tan solo su primer mes de publicación acceder a su tercera edición, y que la novela de Desnoes motivó la producción de la que es considerada la película más importante de la historia de Cuba, Memorias del subdesarrollo (1968), dirigida por Tomás Gutiérrez Alea (con el propio Desnoes como guionista). Respecto de la novela de Carpentier, se trató de la primera obra que editó el por entonces más relevante escritor cubano -y probablemente uno de los principales ya en todo nuestro continente- desde el comienzo de la Revolución, y su recepción fue desde un inicio universal. ${ }^{2}$

\footnotetext{
${ }^{2}$ Circunscribiéndonos a la narrativa, debemos integrar a este listado de obras de trascendencia en el momento de su aparición a Tierra inerme (Alonso, 1961), La búsqueda (Sarusky, 1961), Maestra voluntaria (Olema, 1962), Gente de playa (González Cascorro, 1962), Adire y el tiempo roto (Granados, 1967), Tute de Reyes (Benítez Rojo, 1967), Los niños se despiden (Fernández, 1968), y Sacchario (Cossío, 1970). Las obras de Benítez Rojo y de Fernández ganaron el premio Casa de las Américas, mientras que el resto recibió en el mismo concurso menciones especiales.
} 


\section{Leonardo Martín Candiano}

Cabe destacar, además, que todos estos autores nombrados -a excepción de Carpentier- publicaron por primera vez un libro en Cuba a partir del triunfo de la Revolución, y muchos de ellos no llegaban a los 30 años de edad al momento de la salida de las obras mencionadas. ${ }^{3}$

Tal diversidad de poéticas funcionando simultánea y masivamente en Cuba no fue un mero producto de orientaciones particulares de ciertos escritores, sino que recibió el espaldarazo de instituciones y de políticas editoriales que distinguieron y difundieron tales propuestas convirtiéndolas en potenciales modelos en el marco de la creación de un original movimiento literario nacional.

A partir de lo expuesto, se advierte que las palabras vertidas por los cineastas cubanos en su documento público de 1963 divulgado en La Gaceta de Cuba respecto de la necesidad de una coexistencia de ideas y tendencias estéticas como condición para el desarrollo del arte (Pogolotti, 2006), fue asumido dentro del ambiente literario. Resulta evidente que tal convivencia se convirtió en más de una ocasión en lucha abierta y en debate público, sin excluir concretas situaciones polémicas realizadas coyunturalmente por algunas instituciones, como la actitud de la UNEAC en la edición de su premio nacional de 1968 ante la decisión del jurado de coronar el poemario Fuera de juego de Padilla y la obra teatral Los siete contra Tebas de Arrufat. Algo similar podemos señalar, ya fuera del ámbito literario, respecto de la prohibición del documental PM por parte del ICAIC en 1961, así como del cierre de la editorial El Puente en 1965.

Sin embargo, durante esta trayectoria de más de una década los debates públicos en medios oficiales y la amplitud estética hegemonizaron la práctica revolucionaria. La compilación realizada por Pogolotti en Los polémicos sesenta otorga un muestrario representativo de ello en el lapso 1963-1966, lo que otorgó continuidad al afán polemista que nutrió las páginas del primer magazine cultural que fundó la Revolución Cubana, Lunes de Revolución, desde el que sus referentes se enfrentaron a grupos intelectuales igualmente integrados al proceso, tanto a la ortodoxia comunista agrupada en el periódico Hoy y en el Consejo Nacional de Cultura como a los ex origenistas -cuyo espíritu notaban presente en la Nueva Revista Cubana nacida prácticamente al mismo tiempo que Lunes- y a la dirección del ICAIC (Estupiñán, 2015).

De allí que lejos de notar una homogenización a partir de la instauración del socialismo en Cuba en 1961, Pogolotti postule la existencia de un "policentrismo" institucional en la isla durante los años sesenta que permite comprender la heterogeneidad de la práctica artística en esta etapa, algo semejante a la noción de "soberanía múltiple" que describe Martínez Pérez en el terreno cultural para describir los rasgos identitarios del período. Esto es, la presencia dentro de la

\footnotetext{
${ }^{3}$ Cabrera Infante poseía 30 años cuando se publicó Así en la paz como en la guerra, Edmundo Desnoes y Lisandro Otero tenían igual edad al editarse No hay problema y La situación respectivamente. Reinaldo Arenas contaba con 22 al recibir una mención nacional con Celestino antes del alba, Jesús Díaz y Norberto Fuentes 24 cuando ganaron el premio Casa de las Américas, Barnet 26 cuando salió la primera edición de Biografía de un cimarrón y Heras León 26 cuando logró una mención continental con su primera obra: La guerra tuvo seis nombres.
} 


\section{Escritores en la Revolución Cubana. Articulaciones entre literatura y política en los años sesenta (1964-1966)}

organización de la cultura cubana de variados grupos de acción intelectual que promovieron producciones y acciones diferenciadas dentro del proceso revolucionario, lo que incluyó espacios de formación, orientación, producción, difusión y crítica sostenidos con lineamientos discordantes entre sí.

$\mathrm{Si}$ advertimos que Cabrera Infante ${ }^{4}$ fue el director del popular magazine cultural Lunes de Revolución al momento de la salida de Así en la paz..., que Jesús Díaz lo era de El caimán barbudo meses después de su premiación en Casa de las Américas, que el poeta Roberto Fernández Retamar ocupó ese mismo lugar en Casa desde 1965, que Carpentier comandó la Editorial Nacional de Cuba desde su fundación hasta 1966, que desde 1964 Lisandro Otero se convirtió en el vicepresidente del Consejo Nacional de Cultura -cuya Dirección de Literatura y Publicaciones era ejercida por José Lezama Lima5-; se vislumbra que los escritores detentaron un activo rol no solo en el terreno específicamente literario sino también en la gestión cultural y en la constitución desde ella de un nuevo intelectualismo integrado al proceso revolucionario. Mucho más aún si incluimos en este listado a otros reconocidos autores como Cintio Vitier, Director de la Nueva Revista Cubana e investigador de la Biblioteca Nacional, o Nicolás Guillén, presidente de la UNEAC. De allí que el propio Retamar reconozca que en Cuba fue la propia Revolución la que creó las bases para la existencia de una sociedad intelectual en el país (Dalton et al, 1969).

Este rasgo del proceso intelectual de la isla encontró a mediados de los años sesenta un tiempo de afianzamiento. Señalado como el "cenit de la política independiente del socialismo cubano" (Guanche, 2008: 62), desde 1964/65 hasta 1968 Cuba vivió su auge heterodoxo 6 . Documentos como "El discurso de Argel" y El socialismo y el hombre en Cuba (ambos de 1965) de Ernesto Guevara, la aparición pública del grupo del Departamento de Filosofía de la Universidad de La Habana -a través de su decisión de dejar de utilizar manuales de procedencia soviética en la formación marxista (1964), la fundación de El caimán barbudo (1966), Pensamiento Crítico (1967) y Referencias (1967), y la decisión del liderazgo político de encomendarle la dirección del Instituto Cubano del Libro (1967)-, la organización de la Conferencia Tricontinental (1966), de la OLAS (1967) y el éxito del Congreso de La Habana de 1968, así como el deterioro de la corriente ortodoxa evidenciado en las consecuencias del Caso Marcos (1964), el cierre de las Escuelas de Instrucción Revolucionaria dirigidas por militantes del antiguo Partido Socialista Popular (1967) y la desaparición de las revistas Mella (1965), Cuba Socialista (1966) y Teoría

\footnotetext{
${ }^{4}$ Cabrera Infante fue el único entre los nombrados que abandonó la isla en esta etapa, al autoexiliarse en Europa en 1965, mismo año en el que Arenas recibe un galardón de la UNEAC por su vanguardista obra Celestino antes del alba. Tres años después Cabrera Infante inició públicamente su cuestionamiento a la Revolución con un artículo publicado en la revista argentina Panorama.

${ }^{5}$ Llama la atención que Rafael Rojas pretenda circunscribir la inserción de Lezama Lima en la Revolución Cubana a la redacción de un mínimo número de breves artículos de ocasión, obviando su rol en las estructuras del Consejo Nacional de Cultura, máximo organismo de gobierno en el área.

${ }^{6}$ Como comprobación de la propuesta de Aurelio Alonso sobre las contradicciones que ha tenido el proceso revolucionario, este auge heterodoxo confluyó con la fundación de las UMAP (1964-1967), espacios de reclusión en el que, entre otros sectores, recalaron homosexuales (entre ellos el fundador de Editorial El Puente, José Mario Rodríguez).
} 


\section{Leonardo Martín Candiano}

y Práctica (1967) -todas sostenidas por integrantes del antiguo destacamento político del marxismo influenciado por la URSS-, muestran que la Revolución potenciaba su identidad rebelde y que las palabras del Che al afirmar que la cubana era una revolución contra las oligarquías pero también contra los dogmas revolucionarios se confirmaban a través de prácticas concretas. Como expuso en aquellos años sesenta Edmundo Desnoes: "una de las virtudes de la revolución ha sido dinamitar los esquemas, explorar, inclusive a riesgo de equivocarse, pero nunca corriendo el más terrible de los riesgos: anquilosarse. Y la cultura no es una excepción" (Dalton et al, 1969: 149). Dentro de ese específico tiempo histórico se encuentran los textos que analizaremos a continuación.

\section{Posicionamientos públicos en Casa}

En este contexto, las numerosas publicaciones oficiales cubanas fueron caja de resonancia de una actitud que identificaba a la práctica política, a las ciencias sociales y a la producción artística. Casa de las Américas fue la más relevante, aunque no la única.

La centralidad de esta institución y de su órgano de difusión es ampliamente reconocida hoy día hasta considerarla el principal ícono de la cultura cubana. Quintero Herencia ha destacado que la revista: "puede ser leída como la bitácora pública de un itinerario intelectual en la que se fueron cifrando estrategias de autodefinición de un campo y de una institución cultural latinoamericana” (56). De allí la trascendencia de recuperar las intervenciones que de ella emanan.

En el número doble 22-23 perteneciente a los meses de enero-abril de 1964 la publicación dirigida por Haydeé Santamaría difundió dos documentos que permiten explicitar cómo la intelectualidad cubana pensaba la articulación entre literatura y revolución mientras dirigía las nuevas instituciones estatales, problematizaba la concepción y la función misma del intelectual y producía su obra. Uno de ellos fue "Conversación sobre arte y literatura", versión taquigráfica de una mesa redonda organizada por Casa de las Américas y protagonizada por el novelista Lisandro Otero, el músico Hugo Blanco y los poetas Roberto Fernández Retamar y Luis Suardíaz. El otro texto fue una entrevista colectiva realizada a trece jóvenes escritores.

En la mesa redonda, Otero explica la precaria realidad cultural cubana previa a 1959 y cómo a partir de allí los artistas encontraron un camino para su desarrollo. Si la posibilidad de publicar en Cuba era prácticamente nula hasta la llegada de la Revolución, Retamar afirma que desde entonces lo referente al arte y a la literatura pasó a estar en manos de los escritores y artistas, algo que en concreto desarrollaron las intervenciones de Blanco al referirse a la música y de Otero y Suardíaz en lo concerniente a la política editorial y al rol de los artistas en los organismos en los que desplegaban labores de administración, gestión y promoción de actividades, como por ejemplo la Dirección General de Música, la Editorial Nacional o la editorial de la UNEAC. 


\title{
Escritores en la Revolución Cubana. Articulaciones entre literatura y política en los años sesenta (1964-1966)
}

Pero para estos intelectuales, la especificidad del arte no se resuelve con la integración de artistas en la gestión de la cultura, sino por la manera de enfrentar los problemas que se arrastran en el área. Otero determina que la escasa experiencia estética del pueblo cubano complejiza la producción. Suardíaz, por su parte, rescata el clima polémico y la amplitud que recorre la isla:

\begin{abstract}
"nuestra incipiente industria gráfica ... contempla en este año la edición de literatura de más de un millón de ejemplares, y como decía Lisandro, de autores muy variados que van desde Maquiavelo a Conrad, de Poe a Flaubert, para citar algunos nombres, y que hemos vertido en nuestra Editorial realizaciones que contemplan los latinoamericanos, los universales y los cubanos, y autores técnicos, cuyos libros utilizamos en especialidades, ya que entendemos que la cultura no está supeditada a un país, a una época, a uno u otro escritor, queremos que nuestro pueblo esté en contacto con la cultura universal en todas en todas sus manifestaciones" (1964: 136).
\end{abstract}

Este posicionamiento sobre la cultura universal como patrimonio de toda la humanidad y que por lo tanto la sociedad cubana recién alfabetizada posee el derecho a su acceso, es reforzado por otras intervenciones. Retamar propone que el criterio cultural y educativo de la Revolución es el de difundir y enseñar lo que se considere de mayor calidad: "lo mismo Leonardo que Picasso, lo mismo Maquiavelo que Steinbeck, quienes acaban de ser publicados por la Editorial Nacional en ediciones populares, para las masas" (Retamar, 1964: 132). Ante posturas que cuestionan el hermetismo de determinado arte o el anacronismo de producciones de siglos previos, remarca: "no es una cuestión de que al hombre por naturaleza le sea más fácil el arte de una época que el arte de otra época. Todas las artes son difíciles. Por naturaleza, a nadie le es fácil un arte $\mathrm{u}$ otro; todas suponen una enseñanza" (130). Por lo tanto, si todo supone una enseñanza es preferible educar a partir de las obras de mayor calidad y complejidad existentes, ya que en ellas se obtiene una experiencia estética más acabada. Blanco confirma que estos lineamientos no corresponden solamente a un escritor o a un área cultural de la Revolución, sino que forman parte de una política de Estado. Refiriéndose estrictamente al ámbito musical en el que trabaja, indica que las técnicas no poseen partido político ni patria, y que todas deben ser aprovechadas por el pueblo cubano:

\footnotetext{
"Los compositores que nos dedicamos a la música culta o como quiera llamársele, seguimos trabajando hacia adelante en cuanto al empleo de las nuevas técnicas, sin volver hacia atrás. ... Ahora yo, por ejemplo, estoy componiendo "música concreta", porque nuestros guajiros, nuestros obreros seguramente querrán escucharla algún día. Es decir, que no creemos necesario acudir a técnicas ya caducas basados en el prejuicio de que "eso es lo que le gusta al pueblo". ... Nosotros no creemos que las técnicas musicales tengan partido o patria. Todas ellas, dondequiera que hayan surgido, son patrimonio de la humanidad y están supeditadas y son dóciles a la voluntad del hombre que las maneja" (Blanco, 1964: 134).
} 


\section{Leonardo Martín Candiano}

Blanco rememora la experiencia realizada por la Dirección General de Música al brindar conciertos en granjas colectivas y fábricas. En esos encuentros con obreros y campesinos las piezas de Mozart, Stravisnki y Bach fueron las que más gustaron al ser oídas por primera vez, lo que lo lleva a pensar: "en lo peligroso que resultaría que un grupo de personas, reunidas en un despacho, se pusieran a determinar qué música prefiere el campesino, qué música prefiere el obrero" (133). Esta afirmación no dista de la que hará célebre Ernesto Guevara en El socialismo y el hombre en Cuba un año después, según la cual lo que suele creer un funcionario sobre lo que es capaz de entender el pueblo no es más que lo que el funcionario de turno entiende y no lo que efectivamente el pueblo puede llegar a comprender. Esa falta de audacia que el Che les endilga muchas veces a los propios revolucionarios en el terreno de las ideas es lo que estos intelectuales pretenden zanjar con tales posicionamientos.

Sin embargo, el diálogo entre una elite intelectual ya establecida y un pueblo que recién está alfabetizándose continúa siendo un problema a abordar. Ante ello, Retamar se pregunta qué es escribir para las masas. Si bien considera apropiado realizar tal interrogación, advierte que no resuelve en sí ningún problema, ya que si los sectores populares no saben leer, esgrimir que se escribe para ellos resulta una falsedad: "Yo vuelvo a citar esta idea marxista que me parece excelente: el hombre es, antes que nada, una posibilidad. Nosotros escribimos para la mejor de esas posibilidades" (Retamar, 1964: 132).

Algo semejante expresa Otero. En primer lugar, comprende que revertir el empobrecimiento educacional y de formación cultural en el que el capitalismo sumió al pueblo cubano es uno de los objetivos que la Revolución se propuso. Ante esa necesidad de elevar el nivel formativo general, la producción de obras cada vez más complejas es indispensable para trascender cualquier estancamiento. Se deben promover, por lo tanto, acciones y producciones destinadas a que el pueblo aprecie cada vez en mayor número el arte en sus más variadas formas para restringir la posibilidad de anquilosar el desarrollo de la conciencia -en este caso, estética- y del pensamiento crítico. Ante ello, sugiere difundir distintas tendencias artísticas para que el gusto estético se desarrolle. Esta propuesta se percibe en la práctica editorial y en la política de premiación de obras de esos tiempos, que como mencionamos en el primer apartado incluyeron desde la poética vanguardista de Arenas a la realista de Soler Puig, desde el testimonio de Barnet a lo real maravilloso de Carpentier.

No obstante, Otero considera que para lograrlo se requiere una ligazón entre artista y pueblo, un diálogo que aún está en ciernes y que es imperioso expandir. Por eso la labor del escritor cubano resulta doble: por un lado, producir una obra de la mayor calidad posible; pero, a la vez, realizar otra clase de textos ligados a la divulgación y el combate urgente, lo que requiere de mayor comunicabilidad y de una accesibilidad masiva inmediata. Se trata de dos funciones diversas pero presentadas como convergentes: preparar con su obra al lector futuro y promover un vínculo actual con la incipiente masa de lectores que surge en la isla, lo que deriva en la expansión de los roles del intelectual: 


\title{
Escritores en la Revolución Cubana. Articulaciones entre literatura y política en los años sesenta (1964-1966)
}

\begin{abstract}
"En el caso de los escritores cubanos, se está tratando de hacer una obra de calidad, una obra seria, profesional. Al mismo tiempo, todo escritor cubano realiza otro tipo de trabajo que es una obra de divulgación, de combate, accesible al pueblo al nivel en el cual fue dejado por la burguesía, al nivel que tiene ahora. Eso quiere decir, por ejemplo, en el caso de los poetas, que han creado textos para himnos revolucionarios, cuya música ha sido escrita por compositores sinfónicos. Los dramaturgos, aparte de hacer su obra de calidad, elevando el nivel, han hecho pequeñas obras de un acto, muy sencillas, que pueden ser llevadas al campo y a las industrias. Los pintores, al mismo tiempo que siguen desarrollando su pintura, han hecho afiches revolucionarios y carteles" (Otero, 1964: 132).
\end{abstract}

Por todo lo antedicho, esta mesa redonda ofrece un balance de la situación de la cultura cubana en el primer lustro de la Revolución y las diferencias entre la política de ese momento y la previa al triunfo revolucionario. A su vez, demuestra la integración de escritores y artistas en el andamiaje cultural creado a partir de 1959, y desde ese espacio ofrece la perspectiva de una serie de intelectuales sobre la política a desplegar en el área, que sintéticamente podemos describir como de sostenimiento de una diversidad estética, profundización de la experimentación, democratización de la práctica y de la enseñanza artística y ampliación de las funciones del intelectual. Por último, se busca quebrar el hiato entre elite cultural y pueblo recién alfabetizado, por lo que se aspira a lograr una mayor comunicabilidad que no vaya en detrimento de la experimentación sino que coexista con ella. Así se debe garantizar la heterogeneidad de formas estéticas mientras que el escritor no solo tiene que pensar en su obra, sino también en la difusión de la misma, en la formación del pueblo, en el despliegue de la revolución y en la confluencia de su especificidad escrituraria con otras prácticas en el extenso campo de la construcción del socialismo en un país denominado subdesarrollado.

\section{La voz de la nueva literatura cubana}

En el mismo número, Casa publicó una entrevista colectiva realizada a jóvenes escritores: Humberto Arenal, Calvert Casey, Abelardo Piñeiro, Rogelio Llopis, Luis Agüero, Barnet, Luis Marré, Desnoes, Noel Navarro, Otero, José Lorenzo Fuentes, Fernández Retamar y Sarusky respondieron siete preguntas sobre el vínculo entre práctica estética y Revolución.

En líneas generales, las respuestas muestran homogeneidad, lo cual no resulta extraño debido a que varios de ellos provenían de la experiencia de Lunes de Revolución. Este hecho, además, refuerza la idea de que en Casa encontraron dentro de este sector a aquellos escritores que les resultaban más cercanos en términos estéticos y políticos (Quintero Herencia; Gilman).

Todos coinciden en señalar la importancia de la Revolución en sus proyectos creadores. Sin ella, los interrogados sienten que no existirían dentro del ámbito intelectual. Arenal afirma que fue la Revolución quien le brindó los medios materiales para difundir su obra, ya que por un lado le permitió editar sus textos y 


\section{Leonardo Martín Candiano}

por el otro está creando un público lector. Así, la Revolución le permitió convertirse en escritor profesional al generar las condiciones de posibilidad de un movimiento literario en la isla. Similar respuesta dieron Llopis y Agüero. El primero al indicar que es parte de un grupo de escritores que sin la Revolución aún estarían ansiando publicar un libro; el segundo al reforzar la idea del nuevo receptor de sus obras cuando propone como principal forma en que la Revolución afectó su literatura el hecho de que ahora la gente de Cuba lee los libros que él escribe. Desnoes considera que la Revolución transformó su concepto de la literatura al descubrirle la importancia del otro, en este caso puntual, del lector. Sin bien se diferencia al sostener que este intercambio entre autor y lector no ha madurado lo suficiente, considera que la Revolución logró generar las condiciones necesarias para que eso se realice. Del mismo modo, Retamar esgrime sentirse "más vinculado, más escuchado" (1964: 148) gracias a la Revolución, y Sarusky establece que la antigua "comunicación imposible entre el pueblo y el creador" (1964: 149) ahora es una realidad.

Resulta notorio que la política revolucionaria de fundación de revistas, editoriales, instituciones, premios y concursos, así como el proceso alfabetizador, las escuelas de arte, los movimientos de aficionados, la conformación de la UNEAC y el clima socio-cultural que respiraba la isla, constituían un acervo novedoso y enriquecedor para los escritores cubanos; los cuales, parafraseando a Sartre, empezaban a intuir que el público virtual al que idealmente se dirigían se convertía en un público real al que aspirar.

Otro lineamiento establecido por diversos escritores fue el de considerar que la Revolución provocó una transformación de sus conciencias que conllevó un cambio en sus nociones sobre lo estrictamente literario. Esto es, lejos de cualquier invasión de lo político sobre un presuntamente aséptico campo intelectual -noción presente en la lectura de Rojas y en la de Quintero Herencia-, el advenimiento de un proceso tan raigal como el que estaba desplegándose en Cuba generó originales perspectivas dentro del ámbito literario que debían, por lo tanto, pensarse a partir de sus propia lógica y tradición. Nace entonces un momento de búsquedas y de apuestas por nuevas síntesis, de intenciones de construir un renovado lenguaje literario que plasme la transformación de valores, visión de mundo, mirada sobre el ser humano, experiencias, función social de la práctica intelectual, entre otras.

Que estas consideraciones no fueron pensadas en detrimento de la autonomía de la literatura se advierte en la respuesta de Desnoes, cuando establece: "la revolución nada tiene que ver directamente con la literatura. Son dos fenómenos que existen únicamente remitidos al hombre (...) Es una relación dialéctica cuyo centro de comunicaciones es el hombre" (1964: 145). Casey, por su parte, marcó que la Revolución le ha hecho pensar que los escritores deben hacer la revoluciones desde la literatura, y Llopis que la Revolución le dio la oportunidad de publicar sin tener que encerrarse en una estética determinada.

En otro aspecto en el que hay homogeneidad es en el de certificar la existencia de una lucha generacional al interior de la cultura cubana, matizada por la unidad 


\section{Escritores en la Revolución Cubana. Articulaciones entre literatura y política en los años sesenta (1964-1966)}

dentro del proceso revolucionario de diferentes grupos etarios pero palpitante en todo momento. Teniendo en cuenta que todos los entrevistados son jóvenes, se postulan como quienes han llegado para ocupar el espacio que sus antecesores se niegan a abandonar, por lo que allí radica un conflicto de inevitable desarrollo.

Todos, a su vez, descartan la existencia de temas específicos a los que abocarse, aunque evidencian que los sucesos revolucionarios generan tal conmoción que se tornan muchas veces en contenidos inexorables de sus obras. La Revolución los ha transformado, les dio una misión, diariamente los convoca en sus revistas, instituciones, labores voluntarias en el terreno productivo y militar. La Revolución se funde con sus vidas, y su escritura lo asume.

Coherentemente con lo expuesto sobre la inexistencia de temas específicos, prácticamente ninguno sostiene que el realismo concebido tradicionalmente deba ser la estética de la Revolución; aunque, por lo mismo del vínculo gestado con el proceso en curso, les resulta ineludible problematizar la representación literaria de lo real. Para Arenal, el realismo no es más que un punto de partida, ya que lo importante es lo que el artista hace con la realidad. Casey, Piñeiro y Barnet amplifican el concepto hasta excluirle todo límite. Para el primero: "significa todo, porque lo subjetivo es tan objetivo para el individuo como el mundo exterior" (1964: 140), mientras que para el segundo es: "todo lo que tiene relación con la conciencia o los sentidos, desde un perro hasta el sueño de un borracho" (140). Para Barnet, finalmente: "tan objetivo es un fenómeno de neurosis con sus consecuentes alucinaciones como el conjunto de las motivaciones que incitan la acción del hombre" (143).

En la misma línea, Llopis dirá que: "a menudo la imaginación que inventa mundos, personajes, experiencias o situaciones irreales crea símbolos que ponen al lector en contacto mucho más estrecho con la realidad" (141), y Agüero sostiene: "para el arte puede ser bien real que el revólver de Durango Kid dispare 200 tiros seguidos o que un hombre se pase la vida entera tratando de entrar a un castillo" (142). Dentro de similar tónica, Desnoes sugiere que un escritor comprende el realismo cuando logra descubrir la mayor cantidad de relaciones posibles entre elementos aparentemente caóticos dentro de una experiencia humana. Fuentes dirá que la realidad es infinita al igual que los medios para expresarla, y Sarusky, más allá de admitir la existencia de escuelas realistas y naturalistas, postula que éstas no pueden agotar el problema, ya que: "la realidad no es una mariposa inmóvil clavada en la vitrina de algún coleccionista" (149).

Sin haber formado parte de esta entrevista porque su primera obra se conoce recién un año después, estos lineamientos coinciden con los que expone Arenas en el reportaje que le realiza Barnet y que se publica en La Gaceta de Cuba en 1967. Allí, ante la pregunta irónica sobre si su imaginativa y experimental novela Celestino antes del alba es rigurosamente autobiográfica, el autor responde:

"Sí, en lo que respecta a la aparición de las brujas y los duendes, los primos muertos, el coro de las tías infernales, el acoso de infatigables hachas, los 


\section{Leonardo Martín Candiano}

desplazamientos del personaje hacia la luna (huidas) sin obtener resultados ventajosos. Es autobiográfico también el ambiente, la brutal inocencia con la que se expresan los personajes (...) ¿acaso lo imaginado no es un reflejo de la vida misma?" (Barnet, 1967: 21).

Como se observa, los jóvenes escritores cubanos alientan el carácter dinámico del realismo, abogan por un realismo abierto, plural y multiforme dentro del que se introduce la invención, los sueños y las más inusitadas fantasías, pues éstas forman parte del mismo mundo que los acontecimientos vividos. Un realismo que incluya la experimentalidad en el sentido en el que lo concebía Bertolt Brecht (1984) al vincularlo con los avances formales vanguardistas desarrollados en los años veinte en Europa occidental y al proponerlo desviado de la mímesis, porque una obra literaria no debería disimular el artificio a través del cual se compone. Esa es $s u$ realidad, lo primero que un texto literario no debe falsear.

Particular atención merecen las respuestas sobre la función del escritor en la sociedad revolucionaria. Aquí las perspectivas resultan dispares y se las puede dividir en tres grupos. Por un lado, los asentados en la autonomía literaria restringen la función del escritor a su acto esencial de escritura; por otro, aquellos que integran a la función propia que cataloga su oficio la de participar activamente en la construcción de la nueva sociedad; finalmente, los que sostienen que se debe convertir la escritura en un acto pedagógico y propagandístico al servicio de la Revolución. Así, si un sector se concentra en la renovación estética como expresión de una nueva subjetividad que está naciendo gracias a la Revolución -con lo que se estaría manifestando en la producción literaria la revolución que ya se inició en otros planos sociales-, otro grupo encuentra que el contexto agrega nuevas funciones para el escritor sin que por eso se pierdan las anteriores; posición semejante a la generada en la mesa redonda anteriormente analizada. El tercer sector subsume la labor a lo literario tal como el primero, pero imponiéndole contenidos y orientaciones que no emanan de las necesidades de la obra sino de algo ajeno a ella, como si lo literario sólo pueda contemplar la Revolución, como si la Revolución fuera una exterioridad que a lo sumo se puede alabar, pero de la que se está al margen. Desde esta última perspectiva, pareciera que la transformación socialista la realizaran los dirigentes, los obreros y los campesinos, y que luego el escritor debe llegar para contar eso, para que otros lo conozcan. La cultura se considera, así, un epifenómeno derivado de otra cosa. El escritor será, entonces, el que narre la Revolución que otros hacen.

Particularmente, mientras Desnoes, Agüero, Fuentes y Casey coincidieron en afirmar que el único deber del escritor en la Revolución es escribir; Retamar y Llopis incorporaron a ello la participación en la construcción revolucionaria como cualquier otro ciudadano. Navarro y Piñeiro, sin embargo, notan que la tarea del escritor está ligada a representar la lucha revolucionaria, imponiendo criterios educativos y comunicativos como esenciales. Navarro sostiene que se debe reflejar en la obra a la Revolución que se está viviendo, y Piñeiro que desde el arte se tiene que contribuir a que las masas tomen conciencia de su actuación revolucionaria y a 


\section{Escritores en la Revolución Cubana. Articulaciones entre literatura y política en los años sesenta (1964-1966)}

que se comprenda la revolución. Así, la literatura se convierte en una herramienta secundaria y auxiliar de otras prácticas sociales.

Finalmente, resulta iluminador el listado de escritores que interesan a los entrevistados. Por un lado, la diversidad de autores nombrados muestra la amplitud estética de esta camada de artistas. Por el otro, se observa que en esa variedad se reiteran algunos apellidos que de por sí establecen criterios estéticos de los creadores cubanos. Kafka y Joyce fueron los más nombrados, en seis ocasiones cada uno. Le siguieron Dos Passos y Sartre con cuatro menciones: Proust, Hemingway, Faulkner, Vallejo y Borges obtuvieron tres: Thomas Mann, Henry Miller, Aldoux Huxley, Elliot, Aragón y Neruda dos; mientras que Chejov, Eugene O'neill, Maiacovski, Camus, Hesse, Cendrars, James, Wolfe, Lawrence, Gide, Durrenmatt, Kerouac, Fitzgerald, Chandler, Tenesse Williams, Ginsberg, Cesaire, Eluard, Brecht y Robbe Grillet una.

Esta mera enumeración llamaría la atención de quienes sustentan la teoría de la sovietización de Cuba a partir de 1961 pues, salvo Maiacovski, no existen autores soviéticos aquí. También lo haría el hecho de que se reivindica la literatura norteamericana a la vez que se le endilga una poderosa potencialidad creativa a literatura policial y a la de Ciencia Ficción, comúnmente considerados géneros menores ligados al comercialismo.

La publicación de esta entrevista colectiva muestra que mayoritariamente los jóvenes escritores se orientaban hacia una articulación entre literatura y revolución que no subordinara el primer término ante el segundo, defendiendo la autonomía del quehacer estético sin por ello eludir la nueva responsabilidad del escritor en la construcción revolucionaria. La posibilidad de ensanchar el conocimiento del hombre a partir de experimentaciones estéticas y ofrecerlo al resto de la sociedad era considerada la expresión más cabal de la llegada de la Revolución a la literatura. Sin embargo, esta perspectiva no era absoluta, y hubo quienes analizaron la creación literaria mediante criterios antagónicos a estos, posición que hacia fines de los años sesenta se volverá mucho más influyente hasta convertirse en hegemónica en los setenta.

\section{Los escritores en Bohemia. Literatura y revolución}

Año y medio después, otra entrevista colectiva retornará sobre ejes semejantes desde la revista Bohemia, histórica publicación cubana nacionalizada en 1960.

Esta encuesta giró en torno a la articulación entre literatura y revolución, la representación realista y la realización de un balance de la producción cultural en los siete años pasados desde el triunfo revolucionario. Realizada a doce intelectuales cubanos pertenecientes a diversas camadas -los más veteranos Carpentier, Portuondo y Guillén; los contemporáneos a los líderes revolucionarios Desnoes, Sarusky, Padilla, Alfredo Guevara, Fernández Retamar, Pablo Armando Fernández, Otero y Fornet; más el joven Jesús Díaz, perteneciente a la considerada primera generación formada intelectualmente dentro de la Revolución- ofrece un panorama 


\section{Leonardo Martín Candiano}

abarcador respecto de las posturas que la intelectualidad local iba asumiendo a medida que avanzaba el proceso.

La primera de las preguntas -¿Qué entiende usted por literatura revolucionaria?exhibe posturas encontradas. Guillén y Portuondo sobre todo, pero también Carpentier, cada uno a su modo y con posiciones particulares, sostuvieron criterios semejantes en torno a pensar la práctica literaria revolucionaria como un producto derivado de la acción política insurreccional y del posicionamiento ideológico del autor; mientras que, también con sus matices y divergencias propias, Alfredo Guevara, Otero, Padilla, Fernández Retamar y Sarusky ofrecieron una visión más arraigada en la especificidad estética. Si Guillén afirmó: "la literatura revolucionaria es aquella que denuncia la injusticia económica, sirve a los intereses de quienes son víctimas de esa injusticia y expresa la necesidad de un cambio violento y progresista de las relaciones entre explotados y explotadores" (Guillén, 1966: 22); Portuondo entendió por literatura revolucionaria: "aquella que refleja la nueva realidad revolucionaria y expresa una concepción del mundo marxista-leninista" (1966: 24). Carpentier se inscribió en la misma línea al plantear como tal a: "toda literatura que refleje un proceso revolucionario que haya acontecido realmente" (1966: 22).

En contraposición, una serie de escritores y críticos centró su fundamentación en lo estrictamente estético. Padilla fue quien lo explicitó al establecer que por literatura revolucionaria se debe entender: "La que lleve a cabo transformaciones tan importantes en lo literario como las que una revolución político social produce en cualquier sitio y época" (1966: 26). Para sustentar su postura, niega que el tema revolucionario sea obligatorio al ironizar sobre la obra de Guillén, mucho menos revolucionaria desde su concepción que la de Rimbaud.

Alfredo Guevara -el único entre los doce entrevistados que no era escritor, sino director del Instituto Cubano de Artes e Industrias Cinematográficas- contempló la necesidad del compromiso de la obra con su contexto histórico y social, lo que supone trascender no solo los marcos ideológicos previamente hegemónicos, sino también las concepciones estéticas de tiempos pasados, incorporándolas en una nueva e integral producción. Una literatura revolucionaria debe ser contemporánea a su tiempo, afirmó, y aceptar los riesgos que ello contiene, que son los de encarar la: "ruptura y superación del pasado histórico, de las formas sociales que le representan y de las estructuras ideológicas, inclusive artísticas, que le resultan naturales" (A. Guevara, 1966: 23). No se trata de hacer del arte una propaganda ni hacer una: "apología de la actualidad inmediata, contingente [sino] descubrir la sustancia de nuestra época, y de aproximar, con este sólo acierto, la que prepara su llegada. Esa contemporaneidad tendrá que ser, pues, no sólo ideológica, en un sentido lúcidamente político, sino también, e imprescindiblemente, artística" (A. Guevara, 1966: 23). Similar conclusión ofreció la mirada de Otero al indicar que la literatura revolucionaria debe aportar soluciones originales a los problemas de la creación artística, por lo que tiene que ser novedosa en el terreno formal, a la vez que se orienta a reflejar transformaciones individuales y colectivas en el marco de 


\section{Escritores en la Revolución Cubana. Articulaciones entre literatura y política en los años sesenta (1964-1966)}

un proceso social. De este modo pretende una apretada síntesis entre lo técnico y lo ideológico.

Sobre la contemporaneidad de la obra con su contexto revolucionario se desplegó el pensamiento de Sarusky y, con una tónica más militante, el de Díaz. Este último remarcó que una literatura revolucionaria en Cuba debe tener como universo, como fermento y como asunto a la propia Revolución, y que el creador tiene que sumarse de manera militante a la construcción de la nueva sociedad para así poder narrar desde adentro los sucesos. Pero a la vez, en semejanza a lo expuesto por Otero, Guevara y Sarusky, plantea que debe estar a tono con su contemporaneidad. Como vemos, Díaz también parece buscar un equilibrio entre ambas posturas, pues si por un lado exige la incorporación temática de la Revolución, por el otro agrega la necesidad de contemporaneidad en términos formales, lo que evidencia una crítica al realismo socialista y al tradicional. Sus cuentos de Los años duros en gran medida manifiestan esta propuesta.

Fernández Retamar evadió la discusión contenidista, a la vez que se acercó a la mirada de Díaz respecto de la necesaria militancia del artista a partir de una postura tautológica: "la literatura revolucionaria es aquella realizada por los revolucionarios (25)". Afincada en una perspectiva que matiza esa afirmación se encuentra la postura de Desnoes respecto de que la literatura revolucionaria de Cuba es toda aquella publicada luego del 1 de enero de 1959, incluyendo allí desde la poesía neosurrealista hasta las consignas políticas. Con ello propone que la literatura revolucionaria no sería solamente la que realizan los revolucionarios sino toda la que se expresa dentro de la Revolución, más allá del posicionamiento ideológico de su autor.

Como establece Martínez Pérez, si para unos la revolución estaba en la sociedad y la literatura debía reflejarla, para otros tenía atributos específicamente estéticos, ya sea marcados por rasgos procedimentales o por búsquedas de nuevas formas de acceder a lo real desde el arte. Sin embargo, la diferenciación entre aquellos que privilegiaron la mirada a partir de la obra de arte fue notoria y estableció nuevos posibles espacios de confrontación, algunos de los cuales se desplegaron tiempo después. ${ }^{7}$

Las dos preguntas siguientes de la entrevista establecieron un principio de acuerdo a partir de los que se enfatizaron aspectos diversos. Once encuestados afirmaron la existencia de una literatura revolucionaria en Cuba. El único que la negó fue Díaz. Del mismo modo, la pregunta por el realismo como forma de expresar la realidad revolucionaria no giró alrededor del uso de un estilo mimético o de categorías reflejas, sino que se inscribió en los debates que la representación literaria de lo real ofrecía en los sesenta, desde la eliminación de toda frontera al estilo en que lo proponía Garaudy hasta la adopción del distanciamiento y la experimentación como acercamiento más profundo a lo real.

\footnotetext{
${ }^{7}$ Por ejemplo, durante el debate desarrollado entre la redacción de El caimán barbudo -comandada por Jesús Díaz- y Heberto Padilla respecto de la novela Pasión de Urbino, de Lisandro Otero, y en relación al papel del intelectual dentro de las instituciones estatales en los años 1967-1968.
} 


\section{Leonardo Martín Candiano}

Guillén es quien retomó, precisamente, la propuesta de Garaudy al señalar que: "el realismo es muy amplio, tan amplio que no tiene riberas. Todo es real, todo viene de la realidad. No podemos abolirla" (22). Carpentier, Díaz, Pablo Armando Fernández y Fornet coincidieron en hacer hincapié en la ambigüedad que presenta el término, por lo que toda respuesta se sostiene en la indefinición. El autor de El siglo de las luces se preguntó: “¿por qué usar la palabra realismo en singular? .... El concepto de realismo varía con las épocas" (Carpentier, 1966: 22). Díaz y Fornet repreguntaron en torno a qué entender por realismo. El director de El caimán Barbudo dijo: “¿Qué realismo? ¿Realismo crítico? ¿Realismo socialista? ¿Naturalismo a lo Zola?” (Díaz, 1966: 26), mientras que en Fornet leemos: “¿De qué realismo se trata? ¿Crítico, mágico, socialista, épico o sin riberas?” (23). Pablo Armando Fernández planteó que toda clase de realismo -crítico, objetivo, socialista- puede llegar a convertirse en la forma de representar literariamente la realidad revolucionaria, pero para ello hay que privilegiar su construcción artística y no su fidelidad mimética.

Alfredo Guevara, Sarusky, Otero y Fernández Retamar aprovecharon la pregunta para disputar sentido contra el realismo socialista por un lado y para defender la multiplicidad de tendencias estéticas al interior de la Revolución por el otro, al remarcar que resulta empobrecedor reducir de antemano las posibilidades expresivas. Dijo Sarusky: "la realidad revolucionaria puede expresarse (ya se expresa) de mil formas distintas" (26). El director del ICAIC, por su parte, expresó que el escritor no sólo reseña un acontecimiento sino que fundamentalmente descubre e inventa un mundo nuevo con su obra y genera un producto que antes no existía en la tierra. Por eso: "es creador de nuevas e ilimitadas realidades poéticas, conceptuales, formales" (A. Guevara: 23). Otero expuso: "El hombre y su circunstancia pueden ser expresados de muchas maneras incluso en las que se alejan de su figuración. Para mí el Hombre es lo revolucionario y su expresión debe ser tan infinita y variada como el hombre mismo. Debemos recordar que el surrealismo representó una de las tendencias más revolucionarias de nuestro tiempo" (24). Por último, en Retamar encontramos la siguiente afirmación: "no creo que la expresión de realidad revolucionaria tenga que estar amarrada a ningún ismo estético. Es preferible el consejo de Goethe: ¡Fuego en toda la línea!” (25).

Guevara, Sarusky y Otero, a su vez, discutieron la concepción tradicional de realismo: "Si debemos entender por realismo lo que puede desprenderse de la lectura de ciertos diccionarios y manuales que se autotitulan marxistas o de estética marxista, te diré francamente que no pueden aceptarse estos límites" (A. Guevara: 23). Tales concepciones son para este autor esquemáticas, ajenas a la cultura cubana, y encorsetan la creación. Desnoes lleva tales nociones al límite al cuestionar la pertinencia del realismo en su país: "donde todo se mezcla: la realidad y los sueños, la fantasía y los hechos concretos ... Realismo es un término inventado en otro mundo y para otro mundo que no es el nuestro" (27). Su conclusión principal, no obstante, cuadra dentro de la tónica general: "Los condenados de la tierra deben utilizarlo todo y nunca dejarse oprimir e inmovilizar servilmente por la estética de las sociedades organizadas y estratificadas" (27). 


\section{Escritores en la Revolución Cubana. Articulaciones entre literatura y política en los años sesenta (1964-1966)}

Las últimas preguntas mantuvieron el clima de la encuesta. En la que interroga sobre qué libros cubanos recomendarían a autores extranjeros mayoritariamente se eligió la obra de los líderes de la Revolución o sus influencias históricas, desde los escritos y discursos de Fidel y el Che a los textos de José Martí, manuales de historia de Cuba o relatos testimoniales de la lucha insurreccional, mientras que se diferenciaron en las lecturas a ofrecer a los cubanos, que van desde la obra de Marx, Engels y Lenin (Portuondo) a La Biblia (Pablo Armando Fernández), pasando por Homero (Retamar), el texto sagrado hindú Bhagavad gita (Desnoes) y El Quijote (Fornet). La lista se acrecienta al sumar textos de Camus, Brecht, Malraux, Shakespeare Neruda, Dos Passos, Hemingway, Gorki, Babel y Fannon, entre otros, a los que agregan la propuesta de lectura de autores cubanos contemporáneos como Soler Puig, Díaz, Retamar, Jamís, Otero, Padilla, Baragaño, Desnoes y Guillén.

De este modo se evidencia, por un lado, una mayor concordancia en las respuestas por rango etario -en particular respecto a qué entender como literatura revolucionaria-, a la vez que se sostiene la problematización de la representación literaria de lo real y la necesidad de experimentación formal.

\section{Dentro de la Revolución, todo}

Estos espacios de intervención crítica que hemos analizado comprenden la heterogeneidad como uno de los rasgos identitarios de la práctica intelectual de la Revolución Cubana. Esa diversidad es considerada el motor indispensable para un despliegue cultural que debe ser materializado por una nueva institucionalidad que permita concretar distintos proyectos estéticos.

Por ello este período se caracterizó por la coexistencia y lucha de prácticas culturales discordantes en el marco de un despliegue unitario. De allí que la propagación de encuestas, entrevistas colectivas, polémicas públicas, conversatorios, encuentros y congresos haya sido una práctica frecuente. ${ }^{8}$

Debido a lo expuesto, este trabajo deriva que lo más importante que produjo la Revolución dentro de la literatura en sus primeros años en el poder no haya que buscarlo en una obra, un género o un autor, sino en la promoción de una narrativa nacional, en la búsqueda de novedosos lenguajes literarios, en la reflexión permanente sobre la propia práctica, en su extensísima red editorial y en la génesis de un movimiento literario de masas que formó parte de la creación de un nuevo intelectualismo que revisó la concepción misma del intelectual, su labor y el estatuto social de lo literario dentro de la comunidad de la que emerge.

\footnotetext{
8 A las aquí trabajadas podemos agregar las encuestas colectivas "El papel del intelectual en los movimientos de liberación nacional", "Literatura y Revolución (Los Autores)” y "Literatura y Revolución (Los Críticos)", la transcripción de las mesas redondas "Sobre la penetración intelectual del imperialismo yanqui en América Latina" y "El intelectual y la sociedad", todas publicadas en Casa de las Américas entre 1966 y 1969, en lo que podemos denominar la incorporación de un formato que ya había sido utilizado reiteradamente por Lunes de Revolución desde 1959 y que harán propio también El caimán barbudo, La Gaceta de Cuba y Bohemia en reiteradas ocasiones durante los años sesenta.
} 


\section{Leonardo Martín Candiano}

Más allá de la posibilidad de establecer mediante análisis más específicos la preponderancia de una u otra estética durante la década del sesenta, la Revolución no se ató a ninguna en particular ni generó encorsetamientos para preconcebir la creación literaria, sino que posibilitó dentro de su concepción revolucionaria la emergencia de poéticas diversas, lo cual no excluyó la presencia de agudos debates públicos, más de una querella de envergadura y concretas acciones reñidas con la libertad pregonada que contradicen los lineamientos dominantes. De este modo, forjó una literatura de notoria vitalidad con una prolífica capacidad de producción y difusión. Esta situación catapultó a los narradores cubanos a lugares protagónicos en nuestro continente.

No obstante, si tomamos las obras narrativas mencionadas en el primer apartado de este trabajo y los comentarios de los autores y críticos emanados en los posteriores, se observa que el acontecimiento de la propia Revolución promovió un magnetismo peculiar que hizo predominar la escritura de textos literarios más orientados a asir aspectos ligados a "lo real" desde discrepantes modos de acercamiento estético, que fueron desde la "narrativa de la violencia" hasta el Testimonio, el realismo, el realismo socialista, la literatura comprometida y lo real maravilloso. Pero esto no motivó la clausura de otras formas de entender y practicar el hecho artístico, como se comprueba con la premiación, publicación y difusión de Celestino antes del alba, entre otras. ${ }^{9}$

En estos años sesenta la opción por una poética no eludió la importancia de otras, porque se trataba de concebir una literatura original para lo cual lo necesario era experimentar. La literatura cubana asumía la tradición literaria pero entendía que debía hablar el lenguaje de su tiempo. En el cruce de esa síntesis está gran parte de su riqueza.

Las reflexiones aquí examinadas muestran el interés por integrar al escritor -que mayoritariamente no había formado parte del proceso insurreccional, es decir, desde el asalto al Moncada hasta la toma del poder- a la Revolución mediante una participación activa en la gestión de la cultura y a partir de la gestación de obras literarias innovadoras que expresen, desde la peculiaridad de lo estético, la creatividad y la originalidad que Cuba detentaba en otros aspectos de su construcción político-social. Para ello se consideró imprescindible el debate entre concepciones estéticas diferenciadas y la expansión de la función del escritor hacia otros terrenos (docente, crítico, periodístico, editor, organizador material de un nuevo movimiento cultural), para lo cual, en el marco de una política orientada desde el Estado, el escritor tuvo en sus manos las editoriales, revistas y diversas instituciones. Fue en esta situación que se debatió desde diferentes perspectivas sobre las características de la "literatura revolucionaria", la productividad estética del realismo, la necesidad de experimentación técnica y el vínculo del artista con el resto de la sociedad.

\footnotetext{
9 Podemos agregar aquí Poesía, revolución del ser, de José Álvarez Baragaño, la producción dramática de Virgilio Piñera, la organización del Salón de Mayo en 1967 o el lugar protagónico del músico Juan Blanco en el país, pionero a su vez en la introducción de la música concreta y dodecafónica en la isla.
} 


\section{Escritores en la Revolución Cubana. Articulaciones entre literatura y política en los años sesenta (1964-1966)}

Podemos concluir que así como los escritores demandaron para sí mismos una mayor integración para con la Revolución, también sostuvieron una actitud renovadora en la técnica estética que responde a una responsabilidad específica dentro de su profesión y a una búsqueda por traducir al ámbito literario el quiebre con el pasado. De ese modo buscaron integrarse al proyecto revolucionario.

\section{Bibliografía}

Alonso, A. (2018). "La política cultural de la Revolución Cubana en los 60", Temas $\mathrm{N}^{\circ}$ 95-96, julio diciembre, La Habana. pp. 79-84.

Artaraz, K. (2011). Cuba y la nueva izquierda. Buenos Aires: Capital Intelectual.

Barnet, M. (1967) “Celestino antes y después del alba”, La Gaceta de Cuba, Año 6 N 60, julio-agosto, La Habana. p. 21.

Brecht, B. (1984). El compromiso en literatura y arte. Barcelona: Península.

Casa de las Américas (1964-1966); № 22-23 a º39. La Habana: Casa de las Américas.

Carpentier et. al. (1966) "Literatura revolucionaria” en Bohemia, Año 58, N²9, 22 de julio, La Habana.

Dalton et al (1969). El intelectual y la Sociedad. México: Siglo XXI.

Estupiñán, L. (2015). Lunes, un día de la Revolución Cubana. Buenos Aires: Dunken.

Fernández Retamar, R. (1967) "Hacia una intelectualidad revolucionaria en Cuba", Casa de las Américas $\mathrm{N}^{\circ}$ 40, enero-febrero, La Habana. pp. 4 a 19.

Fornet, A. (2007); Quinquenio Gris: revisitando el término. En Ciclo La política cultural del período revolucionario: Memoria y reflexión. Recuperado de http://www.criterios.es/pdf/fornetquinqueniogris.pdf.

Fornet, J. (2013). El 71. Anatomía de una crisis. La Habana: Letras Cubanas.

Gilman, C. (2003). Entre la pluma y el fusil, debates y dilemas del intelectual revolucionario en América Latina. Buenos Aires: Siglo XXI. 


\section{Leonardo Martín Candiano}

Gilman, C. (2008) Casa de las Américas (1960-1971), un esplendor en dos tiempos. En Altamirano (Dir); Historia de los intelectuales en América Latina I. Los avatares de la ciudad letrada. Madrid: Katz. pp. 259-284

Guanche, J. (2008). El continente de lo posible. Política y cultura en Cuba (1959-1968). La Habana: Ruth editorial.

Guerra \& Maldonado (2009). Historia de la Revolución Cubana. Navarra: Txtalaparta.

Hernández, R. (2009). "El año rojo. Política, sociedad y cultura en 1968”, Revista Estudios Sociales $\mathrm{N}^{\circ} 33$. Bogotá: Universidad de Los Andes. p. 44 a 54.

Kohan, N. (2006). Pensamiento Crítico y el debate por las ciencias sociales en el seno de la revolución cubana. En Crítica y Teoría en el pensamiento social latinoamericano. Buenos Aires: CLACSO. pp. 389 a 435.

Martínez Pérez, L. (2006). Los hijos de Saturno. intelectuales y revolución en Cuba. México: FLACSO-Porrúa.

Pogolotti, G. -COMP- (2006). Polémicas culturales de los 60. La Habana: Letras Cubanas.

Quintero Herencia, J. (2002). Fulguraciones del espacio. Letras e imaginario institucional de la Revolución Cubana (1960-1971). Rosario: Beatriz Viterbo.

Rama, A. (1971). "Una nueva política cultural en Cuba”, Marcha, ํo 49. Montevideo: Marcha. pp. 47 a 68.

Rojas, R. (2006). Tumbas sin sosiego. Revolución, disidencia y exilio del intelectual cubano. Barcelona: Anagrama.

Walsh, R. (1968). “Prólogo”. En Crónicas de Cuba. Buenos Aires: Álvarez.

Recibido: 07/10/2020

Evaluado: $30 / 11 / 2020$

Versión Final: 06/01/2021 